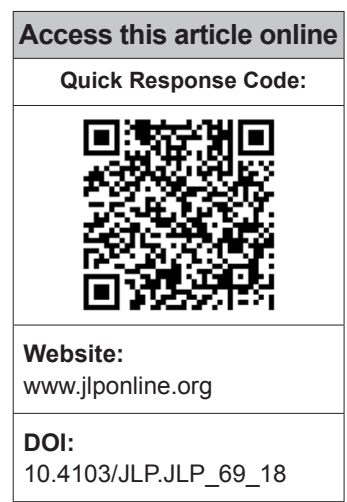

Department of Pathology, PGIMER, Dr. RML Hospital, New Delhi, India

Address for correspondence:

Dr. Prajwala Gupta, Department of Pathology, Room No. 302, $3^{\text {rd }}$ Floor, OPD Building,

Dr. RML Hospital, Baba Kharak Singh Marg, New Delhi - 110 001, India.

E-mail: prajwala2000@ yahoo.com

Submission: 18-05-2018

Accepted: 08-10-2018

\title{
Cytodiagnosis of Rosai-Dorfman disease masquerading as lymphoma: A case report with brief review of literature
}

\author{
Manjari Kishore, Prajwala Gupta, Arvind Ahuja, Minakshi Bhardwaj
}

\section{Abstract:}

Rosai-Dorfman disease (RDD) or sinus histiocytosis with massive lymphadenopathy (SHML) is a self-limiting, benign histiocytic disorder. We report a case of RDD mimicking lymphoma clinically and cytologically in a 3-year-old male child. The present case highlights the importance of cytology in the diagnosis of RDD along with cytological features of other differential diagnoses.

Key words:

Cervical lymphadenopathy, emperipolesis, fine-needle aspiration cytology, sinus histiocytosis with massive lymphadenopathy

\section{Introduction}

Cinus histiocytosis with massive Slymphadenopathy (SHML) or Rosai-Dorfman disease (RDD) is a rare, but well-defined, benign self-limited lymphoproliferative disorder ${ }^{[1]}$ It can be noted in both nodal and extranodal locations. The characteristic cytomorphologic features of RDD are important to be kept in mind as it may sometime mimic either lymphoma or sarcoma. ${ }^{[1,2]}$ Here, we present a case of RDD mimicking lymphoma in a 3-year-old male child who presented with multiple large bilateral cervical lymph node swellings.

\section{Case Report}

A 3-year-old male child presented with bilateral multiple neck swellings for the last 1 year, with infrequent episodes fever, cough, and cold. The patient was apparently well 1 year back following which he developed multiple swellings in the neck. The patient

This is an open access journal, and articles are distributed under the terms of the Creative Commons Attribution-NonCommercial-ShareAlike 4.0 License, which allows others to remix, tweak, and build upon the work non-commercially, as long as appropriate credit is given and the new creations are licensed under the identical terms.

For reprints contact: reprints@medknow.com had an episode of difficulty in breathing. The swelling was insidious in onset and increased gradually. On examination, the bilateral cervical swellings ranged in size from $2 \mathrm{~cm} \times 2 \mathrm{~cm}$ to $5 \mathrm{~cm} \times 5 \mathrm{~cm}$ and had restricted mobility and firm in consistency. There was the absence of any other group of palpable peripheral lymphadenopathy.

There was no history of tuberculosis, loss of weight/appetite, rashes, petechiae or bleeding from any sites, abdominal distension, loose stool, and jaundice. The developmental milestones of the child were normal for the age. The child was vaccinated as per the age. Fine-needle aspiration (FNA) was done twice outside and was reported as Hodgkin's lymphoma.

General examination revealed mild pallor along with multiple large conglomerate lymph nodes in the neck region, ranging in size from $1 \mathrm{~cm} \times 1 \mathrm{~cm}$ to $3.5 \mathrm{~cm} \times 3.5$ $\mathrm{cm}$, fi $\mathrm{rm}$, slightly fixed, and nontender [Figure 1]. Few lymph nodes were also noted in the inguinal region, largest measuring $1 \mathrm{~cm} \times 1 \mathrm{~cm}$. No organomegaly was noted.

How to cite this article: Kishore M, Gupta P, Ahuja A, Bhardwaj M. Cytodiagnosis of Rosai-Dorfman disease masquerading as lymphoma: A case report with brief review of literature. J Lab Physicians 2018;10:460-3. 
Systemic examination did not show any abnormality. Complete blood count along with peripheral smear examination and the biochemical parameters were within normal limits. Ultrasound neck showed multiple enlarged lymph nodes (suggestive of lymphoma), in the neck region at the level 1, 2, and 3 and posterior triangle of the neck at the level 5. Contrast-enhanced computed tomography (CECT) neck showed multiple enlarged lymph nodes of both sides of neck. CECT chest and abdomen showed mild hepatosplenomegaly.

FNA done from bilateral cervical lymph node swellings revealed blood mixed aspirate. Smears prepared were cellular and showed numerous singly scattered histiocytes [Figure 2a]. These histiocytes were larger with abundant foamy cytoplasm, ill-defined cytoplasmic margins, central to eccentrically placed nuclei with marked anisonucleosis, finely granular to coarse chromatin,

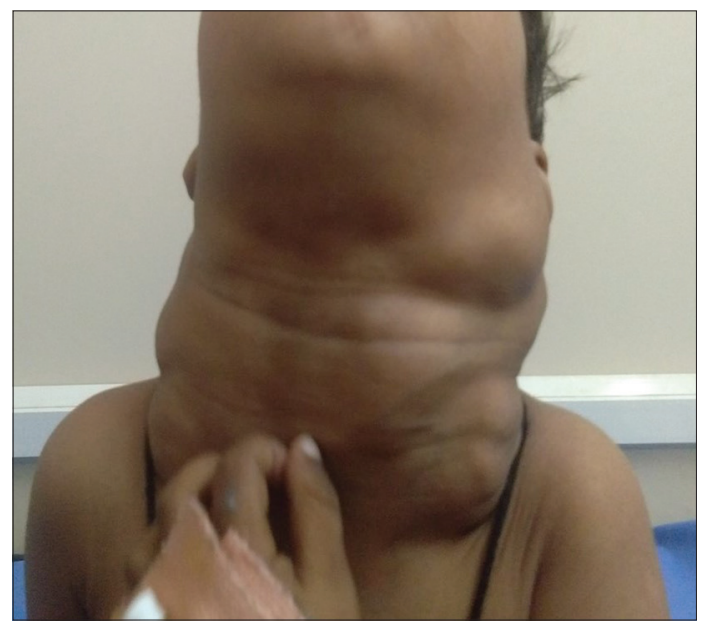

Figure 1: Clinical image showing multiple enlarged cervical lymph nodes and inconspicuous to prominent nucleoli [Figure $2 \mathrm{~b}$ and c]. Many binucleate and multinucleate forms of histiocytes were noted [Figure 2d]. There was evidence of emperipolesis with phagocytosis of lymphocytes and plasma cells at many places [Figure $2 b$ and $c$ ]. Background showed few epithelioid cell clusters, many reactive lymphoid cells, plasma cells, and few eosinophils. ZiehlNeelsen stain for acid-fast bacilli was negative.

A cell block was made from the FNA aspirate and histiocytes showed positivity for CD68 [Figure 2e-f]. Based on the cytomorphological and immunohistochemical examination, a diagnosis of SHML (RDD) was made. The patient further went surgical excision of the lymph nodes which were consistent with RDD. The patient was doing well till the last follow-up few days back.

\section{Discussion}

SHML/RDD is a rare, nonmalignant, histiocytic proliferative disorder. This can occur in a wide span of age from newborn to older age, commonly in the second to third decades. ${ }^{[1,2]}$ There is male preponderance, with a ratio of 3:2. Usually, it is seen in younger individuals, less than 20 years of age, but can affect any age group. It can have nodal as well as extranodal manifestation. The histopathological features of SHML were first described in 1965 by Destombes. ${ }^{[2]}$ Four years later, SHML was recognized as a clinicopathological entity by Rosai and Dorfman. By 1988, as it became evident that other nodal and extranodal sites can also be affected, the term RDD was preferred..$^{[1-3]}$

Classically, it presents as prominent bilateral, painless cervical lymphadenopathy. The common extranodal sites

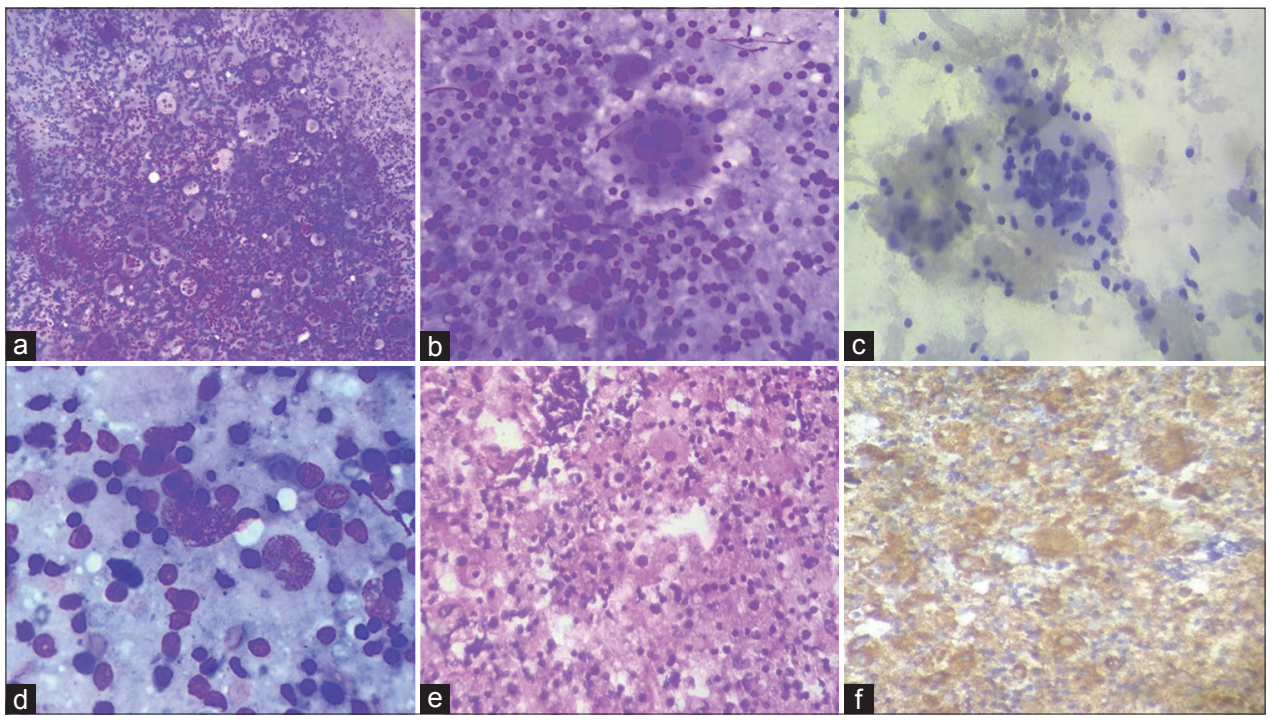

Figure 2: (a) Cellular smear showing numerous singly scattered histiocytes along with reactive lymphoid cells in the background (Giemsa, $\times 100)$. (b and c) Smears showing large histiocytes with abundant foamy cytoplasm, and evidence of emperipolesis (Giemsa, x400; Pap, x400). (d) Binucleate and multinucleate forms of histiocytes were noted (Giemsa, $\times 400)$. Cell block section $(e)(\mathrm{H}$ and $\mathrm{E}, \times 200)$ and immunohistochemistry (CD68) (f) revealing numerous histiocytes 
involved with this disease include the soft tissue, skin, upper respiratory tract, gastrointestinal tract, breast, bones, and central nervous system. ${ }^{[2-4]}$ Clinically, the patient presents with low-grade fever, weight loss, leukocytosis, elevated erythrocyte sedimentation rate, and hypergammaglobulinemia.

Although the etiology of RDD is unknown, two theories have been put forth to describe the pathogenesis of the disease. ${ }^{[3-5]}$ The first theory states that it is caused by a specific infectious process which can be even an acute infection, causing histiocytic proliferation. Second theory states that the disease is due to an aberrant exaggerated immune response to an infectious agent. In our case, the patient was suffering from low-grade fever and mild difficulty in breathing; possibility of the first theory may be relevant.

Cytological examination reveals typically highly cellular smears, many histiocytes with phagocytosed lymphocytes (emperipolesis) in a reactive background of lymphocytes and plasma cells. Emperipolesis is the presence of an intact cell within the cytoplasm of another cell. It can be physiological or pathological. However, it is unlike phagocytosis, in which the engulfed cell is killed by the lysosomal enzymes of macrophage. The engulfed cell remains viable within the other in emperipolesis and can exit at any time without causing structural or functional abnormalities. This is the key morphological feature of RDD. In smear preparations, phagocytosed lymphocytes are not surrounded by a "halo" as they often do in tissue sections which are a fixation artifact.

Sometimes, these histiocytes can be highly pleomorphic revealing large multinucleated cells, mimicking cells of anaplastic large cell lymphoma. However, lack of hyperchromasia, mitosis, and necrosis further aids in excluding a high-grade malignancy. ${ }^{[1,6,7]}$

Immunohistochemistry shows immunoreactivity of histiocytes for S100 and CD68. Although cases have been reported on the histological diagnosis of RDD, Shi et al. presented a case report on cytological characteristics of RDD along with a detailed review of previously published 49 cases on cytology of RDD. ${ }^{[2]}$

Owing to clinical presentations of RDD, it may evoke many differential diagnoses which include Langerhans cell histiocytosis, reactive lymphoid hyperplasia with sinus histiocytosis, hemophagocytic syndrome, lymphoma, and other malignancies. ${ }^{[5-8]}$ Langerhans cell histiocytosis presenting as lymphadenopathy alone is very uncommon. Typically, in this condition, histiocytic nucleus has convoluted shapes with grooves prominent eosinophils being seen. Immunohistochemically, both $\mathrm{S} 100$ and CD1a are positive. Whereas in RDD, histocytes with emperipolesis are very specific, showing positivity for S100 and CD68, and CD1a negative rules out the differential diagnosis of Langerhans cell histiocytosis.

In reactive lymphoid hyperplasia with sinus histiocytosis, there is lack of emperipolesis and negative S100-protein. In hemophagocytic syndrome, other blood cells such as red blood cells, granulocytes, and platelets are seen inside the histiocytes. In contrast, in RDD, the phagocytosed cells are rather lymphocytes; again, emperipolesis is very specific.

It is very important to distinguish lymphoma from RDD, as in smears of lymphoma, monomorphic population of the lymphoid cells is very specific, whereas although polymorphous population of cells is seen in RDD along with typical emperipolesis, still large histiocytes can be confused with Hodgkin's lymphoma or rarely anaplastic large cell lymphoma. In such situations, a detailed evaluation of smears, cell block sections, and immunohistochemistry is warranted to correctly label the specific diagnosis.

Histiocytic sarcoma (malignant histiocytosis) can occur in both nodal and extranodal regions; cytologically, the neoplastic cells show marked pleomorphism, atypia with necrosis, and increased mitotic activity. Emperipolesis can occur in a subset of cases. In RDD, typical cytomorphological picture of emperipolesis in a bland histiocytic background rules out the other malignant association as well. Metastasis from any primary can be excluded by the detailed history and examination of the patient.

With an indolent benign course, RDD does not require aggressive management and regress spontaneously in majority of the cases. ${ }^{[6-8]}$ Recognizing the characteristic cytomorphology and with the help of immunohistochemistry, other invasive and expensive diagnostic procedures can be avoided. However, even if RDD is considered a benign histiocytic disorder that mostly runs an indolent and self-limiting course, a small group of patients may have a more aggressive form of the disease. ${ }^{[6-8]}$ In such cases, nodular, expanding lesions may interfere with the function of vital organs and may eventually be fatal. ${ }^{[8]}$ Our patient also had off and breathing difficulty due to compression of neck structures, with expanding lymph nodes.

\section{Conclusion}

RDD is a rare, inflammatory nonneoplastic process which most often decreases in size without aggressive management. As RDD is infrequently suspected clinically and is a rare disease process, an awareness of the entity along with its clinical profile and its consideration in 
the differential diagnosis in the evaluation of histiocytic and lymphocytic pathologies is essential for proper interpretation and diagnosis of this disease. Although the diagnosis of RDD is made based on clinical suspicion and is confirmed by histopathology and immunohistochemistry, FNA being minimally invasive, cost-effective, and reliable technique can provide a diagnostic clue in the evaluation of this disease process.

\section{Declaration of patient consent}

The authors certify that they have obtained all appropriate patient consent forms. In the form the patient(s) has/ have given his/her/their consent for his/her/their images and other clinical information to be reported in the journal. The patients understand that their names and initials will not be published and due efforts will be made to conceal their identity, but anonymity cannot be guaranteed.

\section{Financial support and sponsorship \\ Nil.}

\section{Conflicts of interest}

There are no conflicts of interest.

\section{References}

1. Rosai J, Dorfman RF. Sinus histiocytosis with massive lymphadenopathy. A newly recognized benign clinicopathological entity. Arch Pathol 1969;87:63-70.

2. Shi Y, Griffin AC, Zhang PJ, Palmer JN, Gupta P. Sinus histiocytosis with massive lymphadenopathy (Rosai-Dorfman disease): A case report and review of 49 cases with fine needle aspiration cytology. Cytojournal 2011;8:3.

3. Deshpande AH, Nayak S, Munshi MM. Cytology of sinus histiocytosis with massive lymphadenopathy (Rosai-Dorfman disease). Diagn Cytopathol 2000;22:181-5.

4. Bist SS, Bisht M, Varshney S, Pathak VP. Rosai Dorfman syndrome with extranodal manifestation. J Assoc Physicians India 2007;55:445-7.

5. Snehalatha R, Senthilnathan R, Ramani P, Herald SJ. The Rosai Dorfman syndrome: The report of a rare case. J Clin Diagn Res 2013;7:1519-21.

6. Vos JA, Abbondanzo SL, Barekman CL, Andriko JW, Miettinen M, Aguilera NS, et al. Histiocytic sarcoma: A study of five cases including the histiocyte marker CD163. Mod Pathol 2005;18:693-704.

7. Li S, Yan Z, Jhala N, Jhala D. Fine needle aspiration diagnosis of Rosai-Dorfman disease in an osteolytic lesion of bone. Cytojournal 2010;7:12.

8. Biswas M, Sarkar T, Das A, Naskar S. Refractory extra nodal Rosai-Dorfman disease of cheek and upper eyelid: A rare case. Ann Pathol Lab Med 2014;1:24-7. 